\title{
Augmented Reality-Systeme zur Unterstützung der Workflow-Ausführung - Entwicklung und praktische Anwendung einer Taxonomie
}

\author{
Johannes Damarowsky $(\mathbb{D}$ - Stephan Kühnel $\mathbb{D}$ - Tobias Seyffarth $\mathbb{D}$ • \\ Stefan Sackmann (iD)
}

Eingegangen: 16. Juli 2021 / Angenommen: 6. Dezember 2021 / Online publiziert: 11. Januar 2022 (C) Der/die Autor(en) 2022

Zusammenfassung Die Ausführung von Workflows ist in vielen Anwendungsfeldern zunehmend mit einer Verarbeitung von Kontextinformationen verbunden. Dies ermöglicht es, den Nutzern die richtige Information zur richtigen Zeit zur Verfügung zu stellen, um die Workflow-Ausführung optimal zu unterstützen. Ein aktueller Ansatz, um eine kontextsensitive Unterstützung zu realisieren sind Augmented Reality-Systeme. Diese verarbeiten Kontextinformationen und liefern notwendige und hilfreiche Workflow-Informationen immersiv und intuitiv, um den Nutzer zu unterstützen und zu entlasten. Da sich die technischen Implementierungen in diesem Feld sehr unterschiedlich ausgestalten, wird in diesem Beitrag eine Taxonomie entwickelt, die es ermöglicht, Augmented Reality-Systeme einheitlich zu systematisieren. Mit dem resultierenden einheitlichen Vokabular bietet die Taxonomie eine praktisch nutzbare Grundlage, um state-of-the-art Augmented Reality-Systeme zu klassifizieren, Trends und Forschungslücken in der Literatur zu identifizieren sowie die Entwicklung neuer Augmented Reality-Systeme methodisch zu unterstützen. Für die Entwicklung der Taxonomie wurden insgesamt 142 Augmented Reality-Systeme analysiert, mit speziellem Hinblick auf die bereitgestellte Workflow-Unterstützung. Die wahrgenommene Nützlichkeit der Taxonomie wurde durch eine Befragung von Augmented Reality-System und Workflow-Management-Experten evaluiert. Zudem werden zwei neuartige Augmented Reality-Systeme, die innovative Ansätze für die Unterstützung der Workflow-Ausführung darstellen, als praktische Beispiele der Anwendung der Taxonomie vorgestellt.

Johannes Damarowsky $(\bowtie) \cdot$ Stephan Kühnel · Tobias Seyffarth · Stefan Sackmann Professur für Wirtschaftsinformatik, insb. Betriebliches Informationsmanagement, Institut für Wirtschaftsinformatik und Operations Research, Martin-Luther-Universität Halle-Wittenberg, Universitätsring 3, 06108 Halle (Saale), Deutschland E-Mail: Johannes.Damarowsky@wiwi.uni-halle.de 
Schlüsselwörter Workflow-Ausführung · Workflow-Management-System · Context-Sensitive Information · Context-Aware Information Systems · Augmented Reality · Taxonomie

\title{
Augmented Reality Systems in Support of Workflow Execution - Development and Practical Application of a Taxonomy
}

\begin{abstract}
The execution of workflows in many application fields is increasingly linked to the processing of context information. This makes it possible to provide users with the right information at the right time in order to optimally support workflow execution. A current approach to realize context-sensitive support are augmented reality systems. These process contextual information and deliver necessary and helpful workflow information immersively and intuitively to support and relieve the user. Since technical implementations in this field vary widely, this paper develops a taxonomy that makes it possible to systematize augmented reality systems in a uniform way. With the resulting unified vocabulary, the taxonomy provides a practically usable basis for classifying state-of-the-art augmented reality systems, identifying trends and research gaps in the literature, and methodically supporting the development of new augmented reality systems. For the development of the taxonomy, a total of 142 augmented reality systems were analyzed, with special regard to the provided workflow support. The perceived usefulness of the taxonomy was evaluated through a survey of experts in the domain of augmented reality systems and workflow management. In addition, two novel augmented reality systems that represent innovative approaches to workflow execution support are presented as practical examples of applications of the taxonomy.
\end{abstract}

Keywords Augmented Reality · Workflow Execution · Workflow Management System · Context-Sensitive Information - Context-Aware Information Systems · Taxonomy

\section{Einleitung}

In vielen Organisationen ist es eine praktische Herausforderung, während der Ausführung von Workflows die richtige Information zur richtigen Zeit am richtigen Ort - also kontextsensitiv - bereitzustellen (Krcmar 2006). Ein derzeit diskutierter und genutzter Ansatz, den damit verbundenen Herausforderung zu begegnen, ist Augmented Reality (AR) (bspw. Blanco-Novoa et al. 2018; Chicaiza et al. 2018; Hofmann et al. 2019; Liebmann et al. 2019; Limbu et al. 2019; Metzger et al. 2018; Wang et al. 2016).

AR erweitert die reale Umgebung um zusätzliche Informationen. Diese synthetischen Objekte werden meist visuell dargestellt, auch wenn grundsätzlich alle Sinne angesprochen werden können. Die synthetischen Objekte reagieren auf die reale Umgebung und passen sich dieser an. AR ist also interaktiv und läuft in Echtzeit ab (Azuma et al. 2001). Sogenannte AR-Systeme (ARS) implementieren AR in Informationssystemen (IS) durch den Einsatz von bspw. Wearables, Headsets, 
Handgeräten oder stationären Systemen (Makris et al. 2013; Metzger et al. 2018; Neges et al. 2015; Wang et al. 2016).

Eine AR-Unterstützung ist natürlich nicht für alle Workflows sinnvoll oder möglich. Während eine Systematisierung sinnvoller Einsatzgebiete für AR derzeit noch intensiv erforscht wird, kann für einzelne Szenarien durch die intuitive und freihändige Bereitstellung kontextsensitiver Informationen mittels AR bereits ein deutlicher Mehrwert aufgezeigt werden. So wurde beispielsweise bei der kollaborativen Planung, Fertigung, Service, Wartung, Lagerkommissionierung, Prozesstraining und -modellierung eine erhöhte Effizienz bei der Workflow-Ausführung festgestellt, die u. a. aus reduzierten Fehlerraten und Ausführungszeiten, einer geringeren kognitiven Belastungen oder weniger benötigtem Training resultiert (Erkoyuncu et al. 2017; Hanson et al. 2017; Hofmann et al. 2019; Jetter et al. 2018; Lampen et al. 2019; Seiger et al. 2021; Wang et al. 2016).

Workflows sind Geschäftsprozesse, welche ganz oder teilweise computergestützt bereitgestellt und von Workflow-Management-Systemen (WfMS) verarbeitet werden. Diese Softwaresysteme ermöglichen die Definition von Workflow-Modellen, interpretieren, instanziieren und verwalten diese, integrieren ggf. externe Anwendungen und ermöglichen Interaktionen mit menschlichen Workflow-Beteiligten (Workflow Management Coalition 1995). Um das Zusammenspiel von AR, Workflows und WfMS systematisch zu charakterisieren und einheitlich beschreiben zu können, bieten sich Taxonomien als Methode an. Eine Taxonomie kann als eine Menge von Dimensionen definiert werden, die jeweils wieder eine Menge von Charakteristiken enthalten, welche zusammen die möglichen Ausprägungen der jeweiligen Dimension erschöpfend darstellen (Nickerson et al. 2013). Durch die Abstraktion der realen Komplexität von Objekten auf diese Dimensionen und Charakteristiken ermöglichen Taxonomien einen einfacheren Vergleich von Ähnlichkeiten und Unterschieden zwischen Objekten. Bei der Analyse von Objektgruppen können aggregierte Ausprägungen der Taxonomie, wie ein häufiges gemeinsames Auftreten oder Fehlen von Charakteristiken, genutzt werden, um zusätzliche Erkenntnisse zu generieren (Bailey 1994). Darüber hinaus tragen Taxonomien zur deskriptiven Wissensbasis bei, indem sie ein Vokabular für eine Domäne und eine Menge von Konstrukten bereitstellen und damit eine Grundlage für zukünftige Forschungsbemühungen schaffen (Hevner et al. 2004; March und Smith 1995).

Aktuell sind nur wenige allgemeine Taxonomien für ARS verfügbar (Kalawsky et al. 2000; Klinker et al. 2018; Milgram und Kishino 1994; Wang et al. 2013). Speziell die Schnittmenge von AR, WfMS und Workflow-Ausführung wird bislang von keiner dieser Arbeiten betrachtet. Das Fehlen eines klar definierten Vokabulars der Begriffe und Konzepte erschwert den Vergleich zwischen verschiedenen ARS und die Identifizierung von Mustern innerhalb der Literatur. Dadurch wird es schwieriger, ARS formal zu charakterisieren, Trends, relevante Literatur und Forschungslücken zu identifizieren sowie neue Forschungsprojekte und Entwicklungen von ARS zu steuern. Um diese Mehrwerte zukünftig zu ermöglichen, wird in diesem Beitrag eine Erweiterung und Aktualisierung der derzeit existierenden Taxonomien vorgenommen, welche die ARS im Hinblick auf ihre Integration mit WfMS charakterisieren und ihre Unterstützung der Workflow-Ausführung detailliert beschreiben. Folglich definieren wir unsere Forschungsfrage: 
Welche Dimensionen und Charakteristiken einer Taxonomie beschreiben aktuelle Augmented-Reality-Systeme zur Unterstützung der Workflow-Ausführung?

Die Entwicklung der Taxonomie erfolgt auf Basis des bekannten Ansatzes zur Entwicklung von Taxonomien nach Nickerson et al. (2013) sowie einer strukturierten Literaturrecherche nach vom Brocke et al. (2009). Bevor in Kapitel 3 der Forschungsansatz detailliert beschrieben wird, werden in Kapitel 2 für eine bessere Anschaulichkeit einige ARS exemplarisch vorgestellt. In Kapitel 4 wird die entwickelte Taxonomie zur Klassifikation von ARS, welche die Workflow-Ausführung unterstützen, mit ihren Kategorien, Dimensionen und Charakteristiken vorgestellt. In Kapitel 5 wird die Taxonomie mittels einer Expertenbefragung evaluiert und in Kapitel 6 die praktische Nützlichkeit und Anwendbarkeit demonstriert, indem Erkenntnisse aus den analysierten 142 ARS und die Entwicklung zweier neuartiger ARS vorgestellt werden. Das Kapitel 7 bietet eine Zusammenfassung dieses Beitrags und einen Ausblick.

\section{Augmented Reality-Systeme in der Praxis}

In der aktuellen Forschungsliteratur wird ein weites Spektrum verschiedener ARS diskutiert, die sich beispielsweise in der verwendeten Hardware, den dargestellten AR-Formaten oder den bereitgestellten Workflow-Unterstützungen unterscheiden. Um diese Bandbreite aufzuzeigen, werden nachfolgend drei exemplarische Implementierungen von ARS vorgestellt.

Das erste Beispiel wird in Blanco-Novoa et al. (2018) vorgestellt. In dieser Arbeit werden Werften durch den Einsatz eines mobilen ARS unterstützt, indem Smartphones zur Erweiterung der Realität um synthetische Inhalte genutzt werden. Mittels eingebauter Kamera können so bspw. visuelle Marker wie QR-Codes erkannt werden, um dann das Kamerabild mit kontextsensitiven synthetischen Inhalten, bspw. Materialbeschreibungen zu überlagern (siehe Abb. 1). Im Kontrast zu mobilen ARS demonstrieren Hou et al. (2013) ein fest installiertes ARS. Hier werden Kameras und Projektoren verwendet, um Nutzer bei der Durchführung einer analogen Bauanleitung durch zusätzliche Inhalte zu unterstützen (Abb. 2). Das vorgestellte ARS

Abb. 1 Augmented RealitySystem. (Aus Blanco-Novoa et al. (2018))

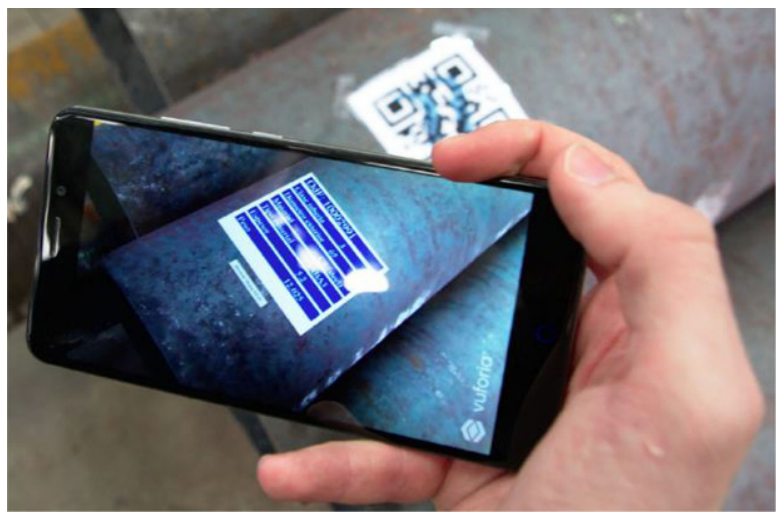



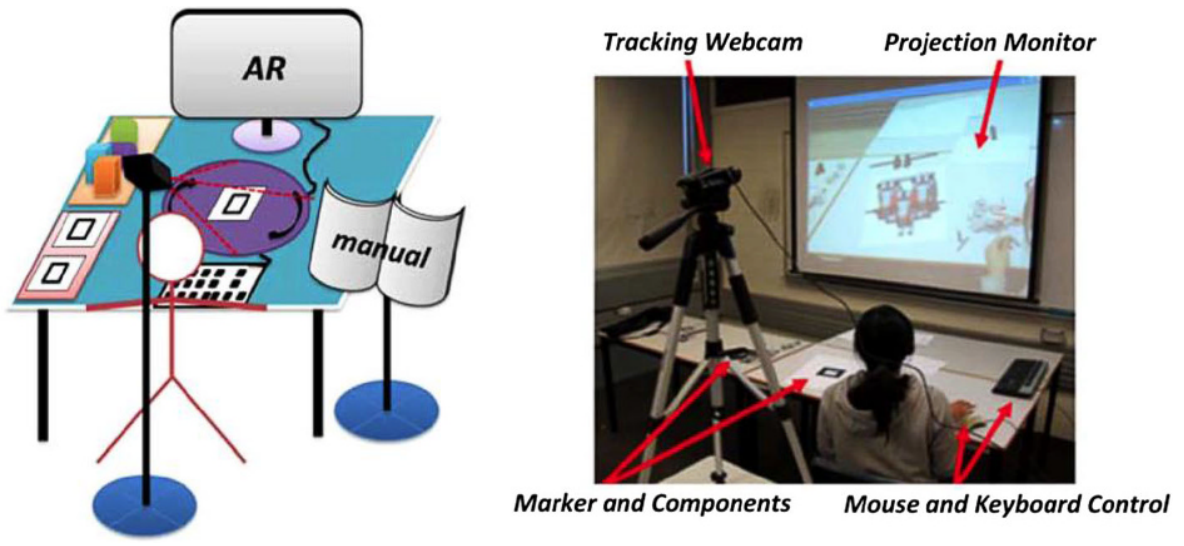

Abb. 2 Augmented Reality-System. (Aus Hou et al. (2013))
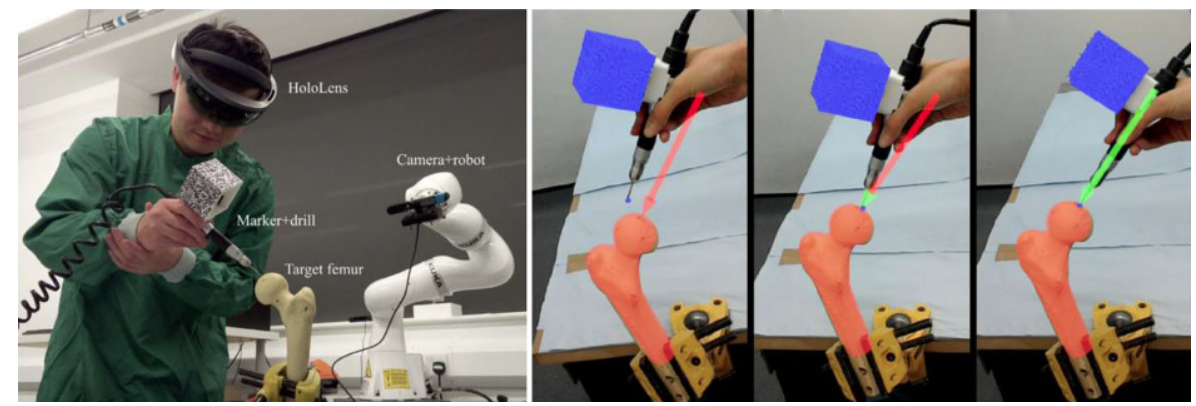

Abb. 3 Augmented Reality-System. (Aus Liu et al. (2018))

erlaubt es dem Nutzer, freihändig zu arbeiten und vermeidet es, zusätzlichen Geräte hinzu zu ziehen oder am Körper zu tragen.

Eine dritte Implementierungsmöglichkeit von ARS zeigen Liu et al. (2018). Für den medizinischen Kontext werden in diesem Beispiel besonders immersiv synthetische Inhalte über ein AR-Headset bereitgestellt. Die Abb. 3 zeigt wie über die Berechnung der relativen Positionen zwischen AR-Headset, des blau markierten Marker-Würfels und des erkannten Knochen-Modells ein präzises Feedback visualisiert wird, ob ein medizinisches Instrument im richtigen Winkel gehalten wird (grün) oder nicht (rot).

Um die drei exemplarischen ARS in einer gemeinsamen Nomenklatur beschreiben und vergleichen zu können, erscheinen bereits einige Dimensionen und Charakteristiken naheliegend. So erscheint der Typ des zur Implementierung genutzten Endgeräts - Smartphone, stationäre Anlage oder AR-Headset - augenscheinlich geeignet. Alle drei ARS nutzen QR-Codes bzw. visuelle Marker, um die synthetischen Inhalte immersiv mit den realen Objekten darzustellen. Auch die Repräsentation der synthetischen Inhalte - Text, Bilder, 2D und 3D Formen - scheint geeignet zum Vergleich. Besonders die bereitgestellte Unterstützung bei der Workflow-Ausführung ist eine interessanteste Dimension für die Praxis. Während das Beispiel in Abb. 1 all- 
gemein hilfreiche Informationen für die Workflow-Ausführung bereitstellt, gibt das ARS in Abb. 2 dem Benutzer, basierend auf den Sensorinformationen, konkrete Anweisungen für die nächste Handlung. In Abb. 3 werden die Sensorinformationen genutzt, um eine Abweichung der aktuellen Ausführung einer Workflow-Aufgabe zu erkennen und dem Benutzer eine präzise, immersive Korrekturanweisung zu vermitteln.

Dieser beispielhafte Vergleich dieser ARS zeigt zum einen deutliche Unterschiede, aber auch erste Ansätze für Dimensionen und Charakteristika einer möglichen Taxonomie auf, um ARS zur Unterstützung der Workflow-Ausführung zu beschreiben. Die Beispiele werfen konsequenterweise aber auch die Fragen auf, welche weiteren relevanten Dimensionen und Charakteristika existieren, um ARS umfänglich zu klassifizieren. Diese Frage wird in den folgenden Kapiteln methodisch untersucht und beantwortet.

\section{Methodischer Ansatz und aktueller Forschungsstand}

Die angewendete Methode zur Entwicklung der Taxonomie nach Nickerson et al. (2013) umfasst fünf zentrale Schritte, welche in Abb. 4 zusammengefasst und im Folgenden erläutert werden.

Der erste Schritt beinhaltet die Definition einer Meta Characteristic, das heißt, eines umfassenden Merkmals auf höchster Abstraktionsebene. Die Meta-Characteristic - in unserem Fall „Eigenschaften von ARS, welche die Workflow-Ausführung unterstützen" - bildet die Grundlage für die Auswahl und Ableitung von Dimensionen sowie spezifischen (Sub-)Charakteristika der späteren Taxonomie.

Der zweite Schritt der Methode umfasst die Definition von Ending Conditions, anhand derer bestimmt wird, wann die resultierende Taxonomie einen zufriedenstellenden Zustand erreicht hat. Dabei werden die von Nickerson et al. vorgeschlagenen 8 objektiven und 5 subjektiven Ending Conditions (OEC, SEC) (vgl. Nickerson et al. 2013, S. 344) angewandt. Die OEC1 fordert, dass alle relevanten Objekte oder eine repräsentative Stichprobe untersucht wurden. Die OEC2 bezieht sich auf die Beschreibung der Objekte durch die Dimensionen und Charakteristika. Werden mehrere Objekte zusammengefasst oder differenziert, muss geprüft werden, ob die Taxonomie angepasst werden sollte. Die OEC3 fordert, dass für jedes definierte Charakteristikum der Taxonomie mindestens ein untersuchtes Objekt vorliegt. Es sollen nicht alle vorstellbaren Charakteristika aufgeführt werden. Die OEC4 definiert, dass die Taxonomie-Entwicklung nicht abgeschlossen sein kann, wenn bei der Untersuchung von Objekten noch neue Dimensionen oder Charakteristika gefunden werden. Die OEC5 fordert das gleiche, jedoch das Zusammenlegen und Aufteilen von Dimensionen und Charakteristika. Die OEC6 und OEC7 fordern, dass die Dimensionen resp. Charakteristika in diesen einzigartig sind und keine Redundanz zwischen diesen vorliegt. Die OEC8 legt dies auch für Kombinationen von Charakteristika fest. Die 5 SEC sind Prägnanz (SEC1), Robustheit (SEC2), Vollständigkeit (SEC3), Erweiterbarkeit (SEC4) und Erklärungskraft (SEC5). SEC1 bezieht sich auf die Anzahl der Dimensionen, die für eine aussagekräftige Taxonomie hinreichend groß sein muss, jedoch nicht zu umfangreich oder überfordernd sein darf. SEC2 zielt 


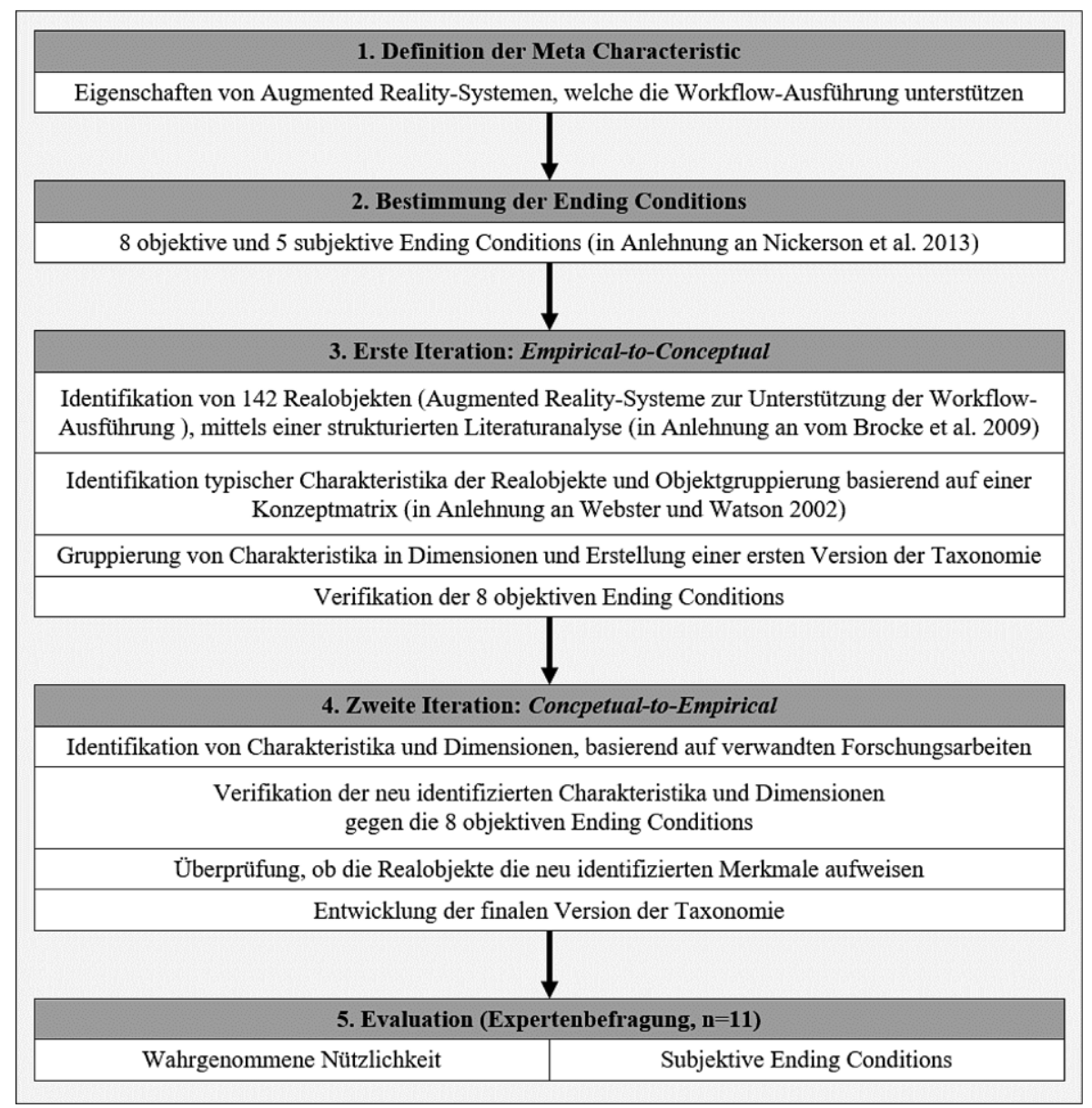

Abb. 4 Forschungsansatz. (In Anlehnung an Nickerson et al. (2013))

darauf ab, dass durch die Dimensionen und Charakteristika der Taxonomie eine ausreichende Differenzierung zwischen Realobjekten möglich sein muss. SEC3 befasst sich mit der Vollständigkeit der Taxonomie, d.h. damit, ob alle relevanten Dimensionen und Charakteristika der Realobjekte auch tatsächlich identifiziert wurden. SEC4 adressiert die zukünftige Erweiterbarkeit bzw. Anpassbarkeit der Taxonomie um weitere Dimensionen und Charakteristika, bspw. wenn neue technologische Entwicklungen es erfordern. SEC5 adressiert schließlich die Erklär- bzw. Aussagekraft der Taxonomie. Dieses Kriterium fordert nicht nur aussagekräftige Erklärungen der Dimensionen und Charakteristika der Taxonomie selbst, sondern auch die Möglichkeit zur Ableitung von erklärendem Wissen über bereits klassifizierte Realobjekte.

Im dritten und vierten Schritt der Methode erfolgt anschließend die iterative Entwicklung der Taxonomie selbst, wofür grundsätzlich zwei Ansätze zur Verfügung stehen: 1) der Empirical-to-Conceptual-Ansatz, bei dem zunächst reale Objekte - im Kontext unserer Studie ARS - identifiziert werden, gefolgt von der Analyse und 
Gruppierung ihrer Dimensionen und Charakteristika, und 2) der Conceptual-to-Empirical-Ansatz, bei dem Dimensionen und Charakteristika zunächst unabhängig von Realobjekten konzeptualisiert werden.

Die Entwicklung der angestrebten Taxonomie wurde im dritten Schritt der Methode in einer ersten Iteration durch den Empirical-to-Conceptual-Ansatz eingeleitet. Nickerson et al. empfehlen hierfür die Durchführung einer Literaturrecherche zur Identifikation von Realobjekten (vgl. Nickerson et al. 2013, S. 345). Dementsprechend wurde hierzu eine strukturierte Literaturrecherche zu relevanten ARS gemäß des Ansatzes von vom Brocke et al. (2009) durchgeführt. Die Abb. 5 bietet einen Überblick über den Ablauf der strukturierten Literaturrecherche.

Um eine möglichst breite Masse an Suchergebnissen zu erhalten, wurden insgesamt fünf Datenbanken durchsucht: ACM Digital Library, EBSCOHost, IEEE Xplore, ScienceDirect und SpringerLink. Der Forschungsfrage folgend, wurden dabei folgende vier Suchbegriffe verwendet: 1. ,augmented reality“, 2. „,system“, 3. ,app“ und 4. ,tool“. Da wir in unserem Beitrag explizit AR untersuchen, haben wir den Begriff „Mixed Reality“ nicht berücksichtigt, da dieser das gesamte RealitätsVirtualitäts-Kontinuums beschreibt und somit eine Obermenge von AR bezeichnet (Milgram und Kishino 1994). Die vier Suchbegriffe wurden mit verschiedenen Suchoperatoren kombiniert und in den Datenbanken getestet. Dabei zeigte sich, dass die einfache Verknüpfung aller Suchbegriffe mit OR-Operatoren zu mehr als 100.000 Ergebnissen führt. Durch die Kombination der Begriffe 2, 3 und 4 mit einem OROperator und die Ergänzung des Begriffs ,,augmented reality“ mit einem ANDOperator wurde eine handhabbare Menge von 3290 Suchergebnissen erreicht. In Anlehnung an vom Brocke et al. 2009 wurden diese Ergebnisse anschließend in fünf Schritten selektiert: 1) Entfernung von Duplikaten, 2) Titel-basierte Selektion, 3) Abstract-basierte Selektion, 4) Volltext-basierte Selektion und 5) Vorwärts- und Rückwärtssuche.

Für die Auswahl wurden vier Inklusionskriterien (IK) definiert, anhand derer über die Relevanz der jeweiligen Artikel entschieden wurde: Um Aktualität zu gewährleisten, wurden nur Artikel berücksichtigt, die nach 2010 veröffentlicht wurden und demnach in zeitlicher Nähe zur Veröffentlichung relevanter Verbraucherprodukte standen, wie beispielsweise der Google Glass im Jahr 2012 oder der Microsoft HoloLens im Jahr 2015 (IK1). Es wurden nur englischsprachige Artikel berücksichtigt (IK2). Artikel, die ARS adressieren, wurden dann als relevant klassifiziert, wenn sie einen vollständigen ARS-Prototypen oder ein vollständiges ARS-Design beinhalten (IK3). Demgemäß wurden keine Artikel berücksichtigt, die lediglich Visionen oder nur einzelne Funktionen von ARS beschreiben. Das beschriebene ARS sollte zudem einen menschenbezogenen Workflow oder einen menschenbezogene WorkflowAufgabe in irgendeiner Weise adressieren (IK4). Es wurden demnach keine ARS berücksichtigt, denen es an einem spezifischen Bezug zur Workflows fehlt, wie bspw. ARS, die für reine Bildungszwecke entwickelt wurden oder ARS, die lediglich ein verbessertes Situationsbewusstsein ermöglichen.

Die resultierende Menge an Literatur umfasste zunächst 60 Artikel, die ARS zur Unterstützung der Workflow-Ausführung beschreiben. Um die angestrebten Dimensionen und Charakteristika relevanter ARS zu identifizieren, wurde eine Autorenzentrierte Analyse durchgeführt, wie sie von Webster und Watson (2002) beschrie- 


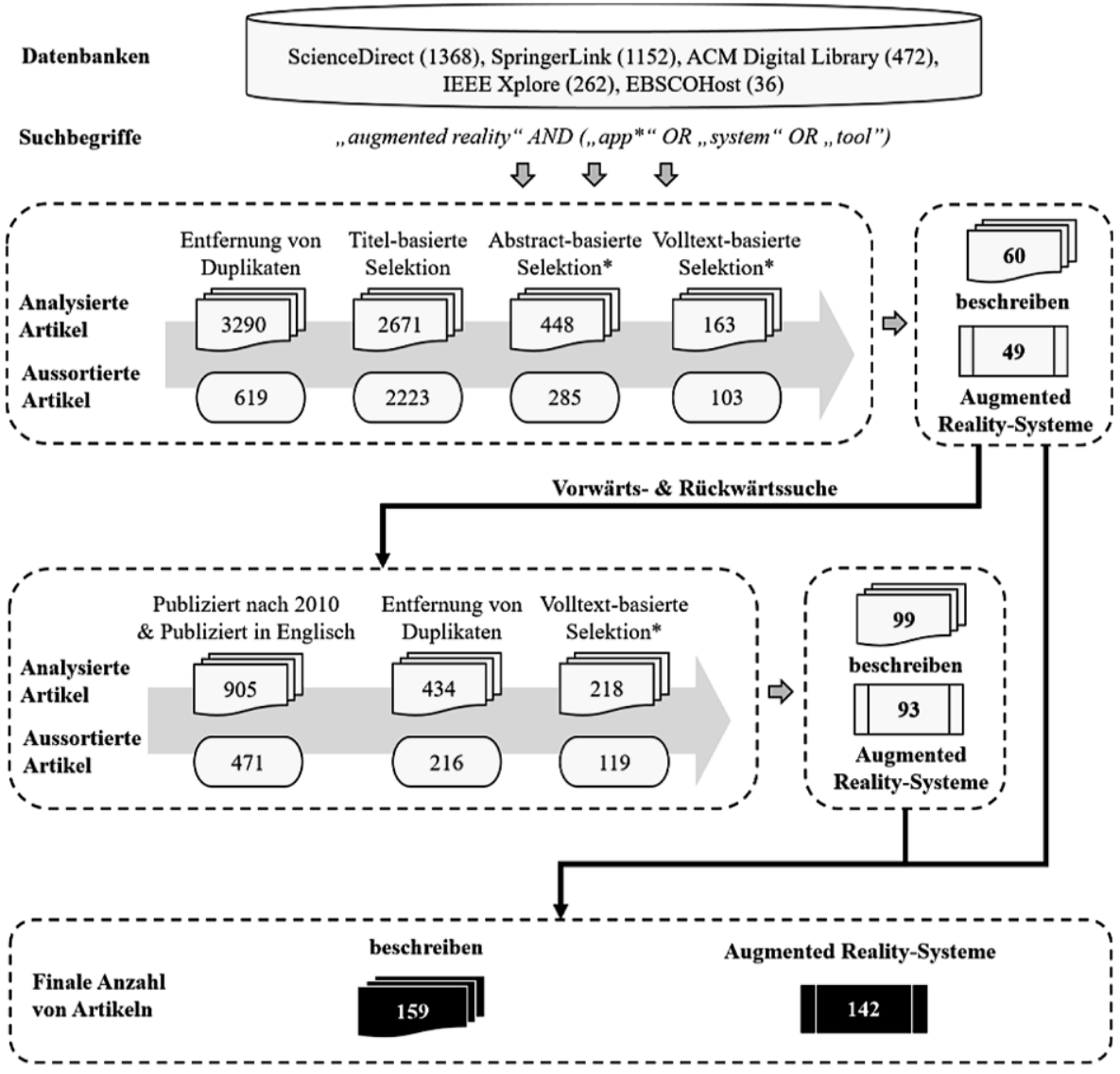
* Inklusionskriterien:
1) Publiziert nach 2010
2) Publiziert in Englisch
3) Adressiert "human workflows" / "human tasks"
4) Vollständige und spezifische Beschreibung

Abb. 5 Prozess und Ergebnisse der strukturierten Literaturrecherche

ben wird. Dabei zeigte sich, dass einige Artikel die gleichen ARS beschreiben, wodurch sich die Menge der zu analysierenden Realobjekte von 60 auf 49 reduzierte. Die anschließende Vorwärts- und Rückwärtssuche brachte insgesamt 905 weitere Ergebnisse hervor, von denen 434 die definierten IK erfüllten. Nach der Entfernung von Duplikaten verblieben 218 Artikel für eine Volltext-basierte Selektion, wobei wiederum 93 relevante ARS identifiziert werden konnten.

Die insgesamt 142 resultierenden ARS wurden schließlich, wie von Webster und Watson (2002) empfohlen, in einer Konzeptmatrix erfasst, anhand derer ihre Charakteristika analysiert und in Dimensionen gruppiert wurden. Die Konzeptmatrix ist im Appendix als Anhang A beigefügt (Onlinematerial 1). 
In Anlehnung an die Ausführungen zu Ex-ante-Evaluationen von Pries-Heje et al. (2008) wurden die 8 objektiven Endbedingungen bereits im Rahmen der ersten Iteration der Taxonomie-Entwicklung berücksichtigt. Darüber hinaus wurde im vierten Schritt der Methode (siehe Abb. 4, Schritt 4) zur Entwicklung einer Taxonomie eine weitere Iteration nach Nickerson et al. (2013) durchgeführt und dabei den Conceptual-to-Empirical-Ansatz implementiert (Nickerson et al. 2013). Ziel war es dabei, die entstehende Taxonomie mit anderen Taxonomien von ARS und Referenzarchitekturen für AR und WfMS zu vergleichen und zu evaluieren, ob bestehende Artefakte möglicherweise noch zusätzliche Charakteristika oder auch widersprüchliche Ergebnisse beinhalten (Kalawsky et al. 2000; Klinker et al. 2018; Limbu et al. 2019; MacWilliams et al. 2004; Milgram und Kishino 1994; van Krevelen und Poelman 2010; Wang et al. 2013; Workflow Management Coalition 1995).

In diesem Zusammenhang wurde insbesondere die von Wang et al. (2013) vorgeschlagene Taxonomie betrachtet, da diese allgemein Implementierungs-relevante Charakteristika von ARS beschreibt und damit eine Teilmenge der angestrebten Taxonomie für ARS zur Unterstützung bei der Workflow-Ausführung ist. Während die Taxonomien im Allgemeinen übereinstimmen, unterscheiden sie sich teilweise im Abstraktionsgrad und in der Formulierung. So bezieht sich die entwickelte Taxonomie bspw. auf „Zweihandgeräte“, während Wang et al. (2013) zwischen „Tablet“ und „Laptop“ unterscheiden. Eine derart feine Aufschlüsselung der Charakteristiken würde die von uns angestrebte Taxonomie jedoch unnötig verkomplizieren und wäre daher nicht mit der OEC2 vereinbar (vgl. Nickerson et al. 2013, S. 344). Außerdem wurden für die entstehende Taxonomie Implementierungs-relevante Charakteristika so definiert, dass sie mit der Arbeit von van Krevelen und Poelman (2010) im Einklang sind, die technische Implementierungsmöglichkeiten von ARS ausführlich beschreibt. Darüber hinaus wurde die entstehende Taxonomie mit dem ID4AR-Framework von Limbu et al. (2019) abgeglichen, welches 11 Arten von synthetischen Handlungsanweisungen beschreibt, allerdings ohne Berücksichtigung von Workflows. Das Framework steht nicht im Widerspruch zu der entwickelten Taxonomie, obwohl ein strukturierter und feingranularer Ansatz gewählt wurde.

Schlussendlich fanden sich in der Literatur auch Charakteristika, die bei der Analyse der 142 ARS bisher nicht identifiziert wurden, wie beispielsweise akustisches und mechanisches Tracking, Geruch und Geschmack als Output oder verschiedene Workflow-Management-Funktionen (Craig 2013; MacWilliams et al. 2004; Milgram und Kishino 1994; Workflow Management Coalition 1995). Entsprechend der OEC3 (vgl. Nickerson et al. 2013, S. 344) wurden diese Merkmale bei der Taxonomie-Entwicklung jedoch nicht berücksichtigt, da sie bisher nicht zur Unterstützung der Workflow-Ausführung eingesetzt werden. Die zweite Iteration des TaxonomieEntwicklungsprozesses bestätigte somit die identifizierten Dimensionen und Charakteristika, sodass die 8 objektiven Endbedingungen als erfüllt angesehen werden können. Die 5 subjektiven Endbedingungen werden hingegen separat im Rahmen der Evaluation der Taxonomie, welche den fünften Schritt der Methode zur Taxonomie-Entwicklung darstellt (siehe Abb. 4, Schritt 5). Dieser wird in Abschnitt 5 beschrieben. Die endgültige Taxonomie als Ergebnis des vierten Schritts der Methode ist in Abb. 6 dargestellt und wird im folgenden Abschnitt 4 erläutert. 


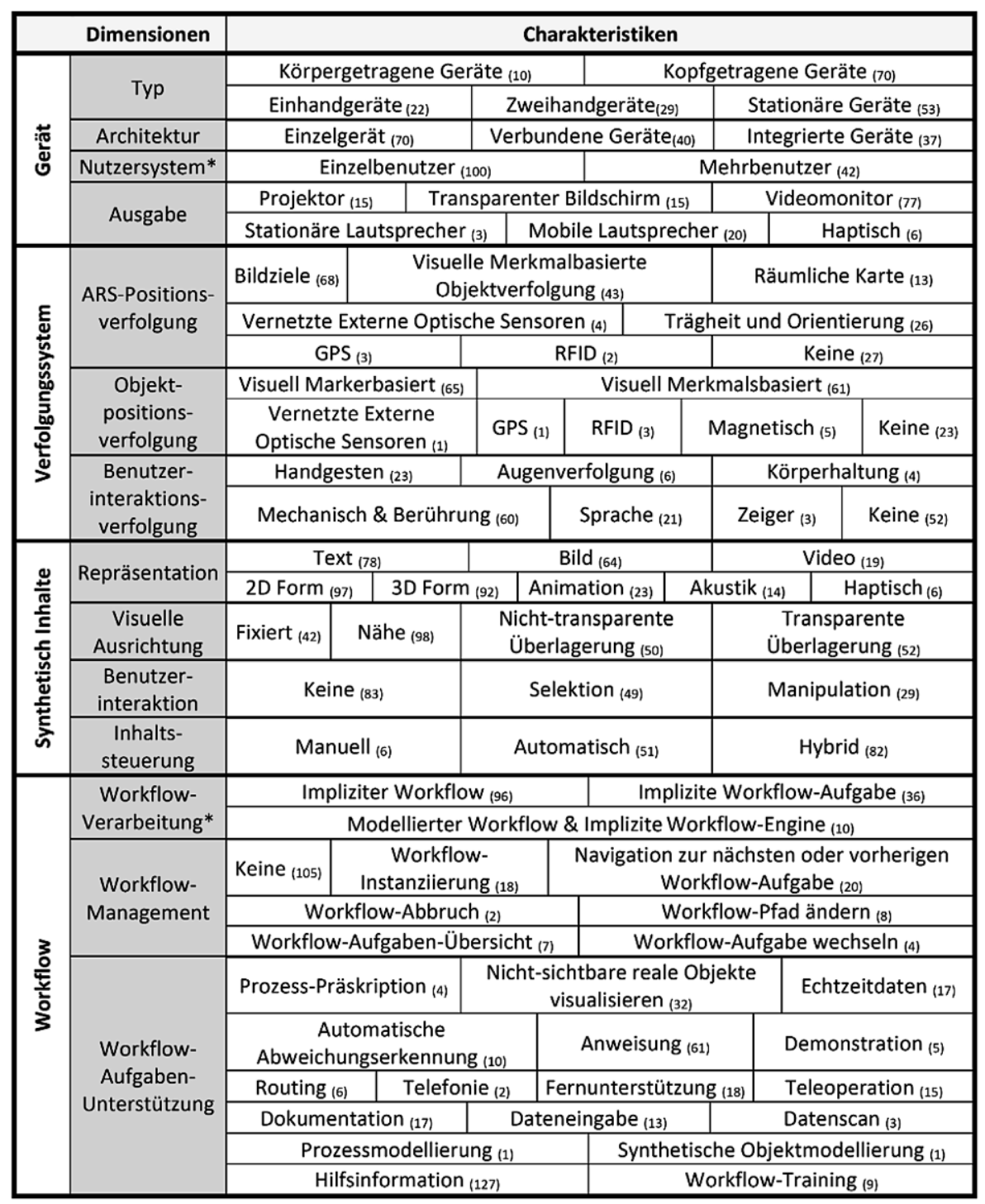

Abb. 6 Taxonomie für Augmented Reality-Systeme zur Unterstützung der Workflow-Ausführung. (* Dimensionen mit exklusiven Charakteristiken, \# Absolute Auftrittshäufigkeit der Charakteristik in den 142 analysierten ARS)

\section{Eine Taxonomie für Augmented Reality-Systeme zur Unterstützung der Workflow-Ausführung}

Da bei der Taxonomie-Entwicklung 14 Dimensionen identifiziert wurden, werden diese der Übersichtlichkeit halber anhand der folgenden vier Kategorien zusammengefasst: 1) Gerät, 2) Tracking-System, 3) Synthetischer Inhalt und 4) Workflow. Die einzelnen Dimensionen werden im Folgenden detailliert beschrieben, die 
identifizierten Charakteristika benannt und anhand der in Kapitel 2 vorgestellten exemplarischen ARS erläutert.

\subsection{Kategorie: Gerät}

In der Kategorie „Gerät“" werden alle Dimensionen zusammengefasst, die die technische Interaktion des Nutzers mit einem ARS charakterisieren. Einige ARS sind mit mehreren Komponenten oder Geräten auf der Seite der Nutzer realisiert, so dass diese ARS mehrere Merkmale in mehreren Dimensionen aufweisen.

Die Dimension ,Typ“ beschreibt die konkreten Geräte, die die Interaktion des Nutzers mit dem ARS ermöglichen. Ein Beispiel für ein Einhandgerät ist das ARS von Blanco-Novoa et al. (2018) (siehe Abschnitt 2), welches auf einem Smartphone umgesetzt wurde. Das ARS von Hou et al. (2013) ist ein Beispiel für Stationäre Geräte. Im medizinischen ARS von Liu et al. (2018) kommt dagegen mit einem ARHeadset ein Kopfgetragenes Gerät zum Einsatz. Auch finden sich in den identifizierten ARS weitere Charakteristika für die Dimension „Typ“, wie Körpergetragene Geräte, die am Körper getragen werden und hauptsächlich als Smartwatches (Wang et al. 2013) realisiert sind und Zweihandgeräte, bspw. Tablets (Neges et al. 2015).

Die Dimension ,Architektur“ beschreibt die physikalische Beschaffenheit eines ARS. Alle Komponenten eines ARS können physikalisch in ein Einzelgerät integriert sein, wie beispielsweise im medizinischen ARS von Liu et al. (2018) in einem AR-Headset oder im ARS von Blanco-Novoa et al. (2018) als Smartphone. Alternativ können die angeschlossenen Geräte physikalisch getrennt aber Verbundene Geräte sein, z. B. Kopfhörer und Smartphone (Aldaz et al. 2015) oder wie dies im stationären ARS von Hou et al. (2013) der Fall ist. Sind die einzelnen Komponenten in einer weiteren Stufe (semi-)permanent verbunden, wird die Architektur als Integriertes Gerät charakterisiert (bspw. Lahanas et al. 2015).

Die exklusive Dimension „Nutzersystem“ beschreibt, wie viele Personen gleichzeitig denselben synthetischen Inhalt wahrnehmen können. Dies kann wie im medizinischen ARS von Liu et al. (2018) ein Einzelbenutzer sein. Alternativ kann das ARS als Mehrbenutzer-System umgesetzt sein, wie bspw. das stationären ARS von Hou et al. (2013) oder das Smartphone-basierte ARS von Blanco-Novoa et al. (2018).

Die Dimension „Ausgabe“ kann zur Informationsvermittlung im Prinzip alle menschlichen Sinne ansprechen. In den in Kapitel 2 vorgestellten Beispielen werden überwiegend visuelle Medien genutzt. Das ARS von Blanco-Novoa et al. (2018) wird auf einem Smartphone implementiert, also einem Videomonitor, der die via Kamera aufgenommene Realität auf einem undurchsichtigen Bildschirm um synthetische Inhalte erweitert. Alternativ kann, wie bspw. beim AR-Headset von Liu et al. (2018), ein Transparenter Bildschirm verwendet werden, welcher vor den Augen des Nutzers angebracht ist und auf welchem die synthetischen Inhalte projiziert werden. Im stationären ARS von Hou et al. (2013) wird wiederum ein Beamer als Projektor verwendet, der Bilder lokal auf Oberflächen und Objekte projiziert. In der Literatur konnten neben visuellen noch akustische und haptische Medien als Ausgaben identifiziert werden. Die akustische Ausgabe kann, wie bspw. bei Fiorentino et al. (2014), entweder über (semi-)fest installierte Stationäre Lautsprecher oder 
wie bspw. bei Chicaiza et al. (2018) mittels Kopfhörer über Mobile Lautsprecher, erfolgen. Die haptische Ausgabe erfolgt bei getragenen Geräten in der Regel über Vibration (Wang et al. 2013).

\subsection{Kategorie: Verfolgungssysteme}

Ein ARS muss weder Objekte noch die eigene Position oder Benutzerinteraktionen verfolgen, um die richtigen synthetischen Inhalte auszuwählen (bspw. Berkemeier et al. 2019; Ferrari et al. 2016; Fiorentino et al. 2014; Liu et al. 2018). Daher werden alle Dimensionen in dieser Kategorie durch „Keine“ ergänzt.

Die Dimension ,ARS-Positionsverfolgung“ beschreibt, wie das ARS seine Position in Bezug auf die Umgebung oder bestimmte Objekte verfolgt, um synthetische Informationen auszuwählen, anzuzeigen oder mit realen Objekten abzugleichen. Viele ARS verfolgen ihre Position nur in Bezug auf Objekte, um synthetische Inhalte in der richtigen Perspektive anzuzeigen, da die relative Position des ARS aus der Verzerrung der verfolgten Bildziele, z. B. QR-Codes oder Fotos, berechnet werden kann, wie bspw. Blanco-Novoa et al. (2018) und Liu et al. (2018) zeigen. Alternativ dazu berechnet die Visuelle Merkmalbasierte Objektverfolgung die relative Position des ARS anhand der bekannten Form eines Objekts (Makris et al. 2013). Auf ähnliche Weise kann ein ARS die lokale Umgebung scannen und eine Räumliche Karte erstellen, um seine eigene Position sowie ,räumliche Anker“ darin zu verfolgen, so dass der Benutzer synthetische Objekte auf jeder realen Oberfläche fixieren kann (Liebmann et al. 2019). Vernetzte externe optische Sensoren, z. B. eine Reihe von Infrarotkameras, können visuelle Marker auf Objekten verfolgen und aus den kombinierten Daten deren räumliche Orientierung ableiten (Henderson und Feiner 2011). Speziell entwickelte Trägheits- und Orientierungssensoren bestehen aus Beschleunigungsmessern, Gyroskopen oder Magnetometern (Liebmann et al. 2019). Positionsinformationen können auch durch den Einsatz netzwerkorientierter Sensoren wie GPS (Dünser et al. 2012) oder RFID-Tags und -Sensoren (Blanco-Novoa et al. 2018) gewonnen werden.

Die Dimension ,Objektpositionsverfolgung“ umfasst ähnliche Merkmale, zeigt sich jedoch bei der Analyse der 142 ARS trotz der Ähnlichkeit unabhängig von der ARS Positionsverfolgung. Es können analog Visuell Markerbasierte und Visuell Merkmalsbasierte Ansätze sowie Vernetzte Externe Optische Sensoren, GPS und $R F I D$ genutzt werden. Zusätzlich können Objekte auch magnetisch verfolgt werden, was insbesondere für medizinische Geräte genutzt wird (Ferrari et al. 2016).

Die Dimension „Benutzerinteraktionsverfolgung“ umfasst vordefinierte Handgesten, die meist über optische Sensoren erkannt werden (Arroyave-Tobón et al. 2015). Die Augenverfolgung misst die Blickrichtung über Pupillenbewegungen oder deren Sichtbarkeit (Aldaz et al. 2015). Auch die allgemeine Körperhaltung des Benutzers kann zur Interaktion genutzt werden, z. B. das Neigen des Kopfes (Aldaz et al. 2015). Mit Hilfe von mechanischen und taktilen Sensoren (z.B. Tasten oder Touchscreen) kann Mechanisch und mittels Berührungen interagiert werden, wie bspw. bei Hou et al. (2013). Mikrofone ermöglichen eine Sprachsteuerung, wie bspw. Blanco-Novoa et al. (2018). Auch Zeiger können zur Interaktion genutzt wer- 
den, z. B. ein Laserpointer, der von den Kameras des ARS verfolgt wird (Andersen et al. 2016).

\subsection{Kategorie: Synthetische Inhalte}

Alle ARS nutzen per Definition eine Form von synthetischen Inhalten.

Die Dimension „Repräsentation“ korreliert zwar mit der obigen Dimension „Ausgabe“, ist jedoch nicht Deckungsgleich. Wir unterscheiden zwischen Text, Bildern, Videos, 2D-Formen, 3D-Formen, Animationen, akustischen und haptischen Repräsentationen (Arroyave-Tobón et al. 2015; Lahanas et al. 2015; Limbu et al. 2019; Metzger et al. 2018; Wang et al. 2016).

Die Dimension ,Visuelle Ausrichtung“ beschreibt die Ausrichtung des visuellen synthetischen Inhalts an die Umgebung aus Sicht des Benutzers. Dieser kann fixiert sein, bspw. wie bei einem Menü in einem AR-Headset oder auf einem Smartphone. Der Inhalt kann in der Nähe eines Objekts oder Markers ausgerichtet sein oder diesen kongruent überlagern, entweder als nicht-transparente Überlagerung oder als transparente Überlagerung (Berkemeier et al. 2019; Makris et al. 2013). Bei Liu et al. (2018) ist bspw. sowohl eine nicht-transparente Überlagerung für das KnochenModell und den QR-Marker dargestellt, als auch der Abweichungsindikator in der Nähe (siehe Abb. 3). In den untersuchten ARS wurden alle akustischen Inhalte nutzerzentriert ausgerichtet, obwohl dies theoretisch auch anders sein könnte.

Die Dimension „Benutzerinteraktion“ beschreibt grob-granular, wie der Benutzer mit dem synthetischen Inhalt während der Ausführung der Workflow-Aufgabe interagiert, entweder gar nicht (keine), wie bspw. bei Liu et al. (2018), durch Selektion von Inhalten (z. B. Menüpunkte bei Arroyave-Tobón et al. 2015) oder durch Manipulation des synthetischen Inhalts, z. B. durch Änderung der Farbe, Form, Skalierung, Position, Orientierung oder Einfügen und Löschen von Objekten (ArroyaveTobón et al. 2015).

Die Dimension ,Inhaltssteuerung“ gibt an, wie der synthetische Inhalt während der Ausführung der Workflow-Aufgabe instanziiert wird. Bei einer automatischen Steuerung werden die synthetischen Inhalte ohne Zutun des Nutzers dargestellt. Ein hybrider Ansatz benötigt gelegentlich eine Nutzereingabe, bspw. in Menüs. Die manuelle Steuerung benötigt für jede Änderung im dargestellten synthetischen Inhalt eine Nutzersteuerung (Arroyave-Tobón et al. 2015).

\subsection{Kategorie: Workflow}

In dieser Kategorie betrachten wir die ARS aus der Perspektive von Workflows und WfMS. Wir orientieren uns an der Definiton der Workflow Management Coalition (1995) und verstehen Workflows als Geschäftsprozess, welche ganz oder teilweise computergestützt bereitgestellt und von WfMS verarbeitet werden. Ein WfMS ist daher ein Software-System und ermöglicht als solches die Definition, Interpretation, Instanziierung, Verwaltung und das Management von Workflow-Modellen, ermöglicht die Integration von externen Anwendungen und bietet eine Benutzeroberfläche für menschliche Workflow-Beteiligte. (Workflow Management Coalition 1995). In Anbetracht des Funktionsumfangs aktueller WfMS (bspw. Camunda oder Signa- 
vio) sowie dem genutzten de facto Standard für Workflowmodellierung BPMN, adressieren WfMS und insb. deren Management-Funktionen - in unserer Perspektive - sowohl stellenbezogene Workflows, welche von einer Personalstelle an einem Arbeitsplatz ausgeführt werden, als auch stellenübergreifende Workflows. Für die Erstellung der Taxonomie wurden die ARS speziell aus der Perspektive der Unterstützung der Workflow-Ausführung betrachtet. Dabei verfolgen wir einen breiten Ansatz und schließen einige Randfälle der üblichen Definitionen von Workflows und Workflow-Management-Systemen (WfMS) ein.

Die exklusive Dimension ,Workflow-Verarbeitung“ beschreibt die formale Darstellung und Verarbeitung von Workflows. Ein Impliziter Workflow liegt vor, wenn die Abfolge der von einem ARS präsentierten synthetischen Inhalte durch logische Stufen, Phasen, Auslöser oder Bedingungen strukturiert ist, d.h. nicht immer alle synthetischen Inhalte gleichzeitig präsentiert werden. Beispielsweise sind bei Hou et al. (2013) die synthetischen Anweisungen in einem Workflow geordnet und werden je nach Fortschritt im Abarbeiten der Bauanleitung sichtbar (siehe Abb. 2). Bei Metzger et al. (2018) werden Sprachbefehle eingesetzt, um durch den impliziten Workflow zu navigieren. Im Kontrast dazu werden in einer Impliziten Workflow-Aufgabe immer alle Inhalte gleichzeitig präsentiert. Bspw. werden bei Ferrari et al. (2016) während einer chirurgischen Workflow-Aufgabe immer alle medizinische Instrumente visualisiert. Ein modellierter Workflow beschreibt die Struktur der synthetischen Inhalte in einer formalisierten Notation, z. B. Petri-Netze oder XML-Schemata (Neges et al. 2015). In diesem Fall verwendet das ARS einen Modellierten Workflow \& Implizite Workflow-Engine, die das Modell interpretiert und die Darstellung der entsprechenden synthetischen Inhalte steuert (Neges et al. 2015). Allerdings wurde in keinem der untersuchten 142 ARS eine WorkflowEngine oder ein WfMS explizit erwähnt.

Die Dimension ,Workflow-Management" beschreibt die Möglichkeiten des Benutzers, Workflow-Instanzen zu steuern und zu verwalten. Diese Dimension orientiert sich am Interface 5 „Administration \& Monitoring Tools“ sowie Interface 2 „Workflow Client Applications“ des WfMS Referenzmodels der Workflow Management Coalition (1995). Das Interface 1 „Process Definition Tools“ ordnen wir in die nachfolgende Dimension ein. Die meisten analysierten ARS bieten keine Möglichkeit für das Workflow-Management an. Einige ARS erlauben dem Benutzer eine gezielte Workflow-Instanziierung (Chicaiza et al. 2018), die Navigation Zur Nächsten Oder Vorherigen Workflow-Aufgabe (Berkemeier et al. 2019) oder einen Workflow-Abbruch der laufenden Instanz (Chicaiza et al. 2018), z. B. über ein visuelles Menü. Wenn der Workflow verzweigt ist, können einige ARS basierend auf den Eingaben des Benutzers den Workflow-Pfad Ändern (Neges et al. 2015). Einige ARS können mehrere aktive Aufgaben verwalten und dem Benutzer eine WorkflowAufgaben-Übersicht bereitstellen, d.h. laufende und/oder zugewiesene Aufgaben anzeigen (Evans et al. 2017). Mit manchen ARS kann ein Nutzer die Workflow-Aufgaben wechseln, d.h. eine Aufgabe aus einer anderen Workflow-Instanz auszuführen (Evans et al. 2017).

Die Dimension ,Workflow-Aufgaben-Unterstützung“ gibt an, wie synthetische Inhalte den Benutzer bei der Workflow-Ausführung unterstützen. Mittels ProzessPräskription kann die nächste, beste Aufgabe ausgewählt werden, welche die Ziele 
des Workflows realisiert. Dies erfordert eine komplexe Verarbeitung des relevanten Kontextes, um auf die nächstbeste Aufgabe zu schließen, was normalerweise eine Form der Prozessvorhersage und/oder Prozesssimulation beinhaltet (Gröger et al. 2014). In Makris et al. (2013) beispielsweise unterstützt ein ARS einen MaschinenDemontage-Workflow, indem es die möglichen Bewegungen von Teilen simuliert und dann das nächstbeste zu entfernende Teil anhand der Anzahl der möglichen Bewegungen auswählt und anschließend visualisiert.

Eine passive Workflow-Führung wird durch die Visualisierung von nicht sichtbaren realen Objekten bereitgestellt, z. B. bei visuell verdeckten, aber magnetisch verfolgten medizinische Instrumente während einer Operation (Ferrari et al. 2016). Auch Echtzeitdaten können Orientierung bieten, z. B. Daten von Handmessgeräten oder Datenbankabfragen (Liebmann et al. 2019). Solche Daten können auch die Ergebnisse einer automatischen Abweichungserkennung darstellen, die erkennt, ob eine Aufgabe im Arbeitsablauf falsch ausgeführt wird, noch nicht abgeschlossen ist oder fehlerhaft abgeschlossen wurde. Dies ist beispielsweise bei Liu et al. (2018) umgesetzt.

Eine aktivere Führung erfolgt durch Anweisungen, die dem Benutzer signalisieren, was zu tun ist und eine visuelle Anleitung zur Durchführung einer Aufgabe oder eines Handgriffs beinhalten kann (Berkemeier et al. 2019). In einer Demonstration ist diese Anleitung kontextspezifisch und animiert, z. B. zeigt sie, wo ein Schraubenschlüssel angesetzt und wie er gedreht werden muss, um eine bestimmte Schraube einzuschrauben (Fiorentino et al. 2014).

Ähnlich kontextspezifisch in Bezug auf den realen Standort eines ARS ist das dynamische Routing eines Benutzers zu einem bestimmten Ort (Dünser et al. 2012).

Für kollaborative Formen der Führung von Arbeitsabläufen können Experten per Telefon (Berkemeier et al. 2019) oder Fernunterstützung hinzugezogen werden, welche die Videotelefonie erweitert, indem sie gleichzeitig Zugriff auf das Sichtfeld des Benutzers gewährt und es den entfernten Experten ermöglicht, anleitende synthetische Inhalte zu erstellen, z. B. Annotationen im Sichtfeld des Benutzers zu zeichnen (Limbu et al. 2019).

Ein ARS kann neue AR-basierte Wege zur Ausführung von Workflow-Aufgaben ermöglichen. Teleoperation ermöglicht die AR-gestützte Programmierung, Steuerung und allgemeine Interaktion mit Maschinen oder Robotern (Andersen et al. 2016).

Die Sensordaten des ARS während einer Workflow-Aufgabe können als Dokumentation aufgezeichnet werden, z. B. in Form von Videos des Sichtfeldes des Benutzers (Chicaiza et al. 2018). Über die Verwendung von Navigations- und Verwaltungsmenüs hinaus kann der Benutzer eine manuelle Dateneingabe über Texteingabe, Spracherkennung oder Multiple-Choice-Auswahl vornehmen (Neges et al. 2015). Eine Variante davon ist ein Datenscan, bei dem der Benutzer die Sensoren des ARS auf eine bestimmte Art und Weise ansteuert, um bestimmte Daten zu erfassen, z. B. durch das Scannen eines Barcodes (Feng et al. 2014).

Die Prozessmodellierung umfasst eine Menge spezieller synthetischer Inhalte und Funktionen, um einen Arbeitsablauf oder Prozess in einem formalen Modell zu dokumentieren, z. B. in der Notation der Ereignisgesteuerten Prozesskette (EPK) (Metzger et al. 2018). 
In ähnlicher Weise beschreibt die synthetische Objektmodellierung das Modellieren, Konstruieren, Stapeln, Montieren, etc. von synthetischen Objekten, beispielsweise während eines Computer-Aided-Design (CAD)-Workflows (Arroyave-Tobón et al. 2015).

Alle unterstützenden synthetischen Inhalte, die sich nicht genauer in die oben genannten Merkmale einordnen lassen, werden als Hilfsinformationen bezeichnet, z. B. das Hervorheben interessanter realer Objekte (Evans et al. 2017). Während schließlich jede Workflow-Ausführung als Trainingsinstrument dienen kann und viele ARS in Demonstrationsszenarien entwickelt und getestet werden, bezieht sich das Workflow-Training auf spezielle Arten von synthetischen Inhalten und Funktionen, die das Training explizit erleichtern sollen oder explizit für Trainingsszenarien entwickelt werden, z. B. wenn der Benutzer eine Trainingsaufgabe wiederholen muss, bis eine bestimmte Ausführungsgeschwindigkeit erreicht ist, oder wenn ein Workflow auf einem virtuellen Trainingsobjekt ausgeführt wird (Lahanas et al. 2015).

\section{Evaluation der Taxonomie}

Unsere Evaluationsstrategie umfasst insgesamt drei Schritte: 1) eine Ex-ante-Evaluation der OEC, 2) eine Ex-post-Evaluation der SEC und 3) eine summative Evaluation der wahrgenommenen Nützlichkeit unserer Taxonomie.

In Anlehnung an Pries-Heje et al. (2008) wurde die Ex-ante-Evaluation der OEC bereits im Rahmen der zwei Iterationen unseres Taxonomie-Entwicklungsprozesses durchgeführt. Zusätzlich heben Nickerson et al. (2013) auch die Bedeutung der SEC besonders hervor (vgl. Abschnitt 2). Um eine sinnvolle Beurteilung dieser Aspekte zu ermöglichen und um darüber hinaus eine summative Bewertung der wahrgenommenen Nützlichkeit unserer Taxonomie zu realisieren, haben wir eine Expertenbefragung durchgeführt. Die Bewertung der wahrgenommenen Nützlichkeit wird von Nickerson et al. (2013) auch direkt für summative Evaluationen von Taxonomien empfohlen und ist in diesem Kontext eines der am häufigsten verwendeten Evaluationskriterien (Nickerson et al. 2013; Szopinski et al. 2019). Da die wahrgenommene Nützlichkeit ein nicht direkt messbares Konstrukt ist, haben wir für die summative Evaluation auf die sechs bekannten Skalen-Items (si) von Davis (1989) zurückgegriffen: Schnelligkeit (SI1), Performanz (SI2), Produktivität (SI3), Effektivität (SI4), Einfachheit (SI5), und Gesamtnutzen (SI6). Wir haben sowohl die SEC als auch die SI für unseren Anwendungskontext spezifiziert, d.h. für eine Taxonomie von ARS zur Unterstützung der Workflow-Ausführung (SEC) und für die Anwendung der Taxonomie zur Aufgabenbewältigung (SI), z.B. zur Auswahl von ARS.

Der initial erstellte Fragebogen enthielt einen einleitenden Text über das Forschungsprojekt, die Taxonomie selbst, eine kurze Erläuterung jeder Dimension und Charakteristik sowie zugehörige Fragen/Aussagen zu den SEC und SI. Für die Datenerhebung verwendeten wir intervallskalierte verbal-numerische 7-Punkt-LikertSkalen $(1=$ Stimme gar nicht zu, ..., $7=$ Stimme stark zu). In einem Pretest stellten wir den initialen Fragebogen fünf erfahrenen Testpersonen zur Verfügung und erhielten die Rückmeldung, dass zusätzliche Informationen zur Taxonomie und zum 
Nutzungskontext für die Beantwortung hilfreich sein könnten. Daher ergänzten wir den Fragebogen mit kurzen Beispielen und einem umfangreichen Handout zur Erklärung der Taxonomie. Zur Nachvollziehbarkeit ist der endgültige Fragebogen in Anhang B enthalten.

Bei der Wahl der Stichprobengröße haben wir uns an der sogenannten ,10 \pm 2 Regel“" (Hwang und Salvendy 2010) orientiert, die besagt, dass 8 bis 12 Befragte für Evaluationen der Nützlichkeit ausreichend sind. Ausgehend von einer erwarteten Rücklaufquote von $50 \%$ haben wir den Fragebogen an insgesamt 24 Experten verschickt und 11 ausgefüllte Fragebögen zurückerhalten (tatsächliche Rücklaufquote: $46 \%$ ). Zu den Befragten gehörten ein Senior Manager, ein IT-Projektmanager, ein AR-Ingenieur, ein Software \& Solution Engineer, ein Multimedia-Designer, ein Softwareentwickler, zwei Forschungsleiter und drei wissenschaftliche Mitarbeiter, die sich in Praxis bzw. Forschung alle mit ARS und Workflows befassen. Von den Experten arbeiten 6 in großen, 3 in mittleren und 2 in Kleinstunternehmen/ Organisationen.

Die Abb. 7 zeigt die Ergebnisse der Expertenbefragung in Form von Boxplots, wobei die Bewertungsergebnisse der SEC in Abb. 7a dargestellt sind (Abb. 7a). Der zugrundeliegende Datensatz ist in Anhang C zu finden. Der Median (m) der Ergebnisse für SEC1, SEC2, SEC3 und SEC5 ist jeweils $\mathrm{m}=6$, der Median der Ergebnisse für SEC4 ist sogar $\mathrm{m}=7$. Bei der Auswertung von SEC1 und SEC3 zeigen sich Ausreißer mit dem Wert 4 (teilweise Übereinstimmung). Die Gesamtergebnisse zeigen jedoch ein sehr hohes Maß an Zustimmung, was darauf hindeutet, dass die subjektiven Endbedingungen als erfüllt angesehen werden können. Folglich kann unsere Taxonomie als prägnant, robust, vollständig, erweiterbar und erklärend angesehen werden.

Bezüglich der Skalen-Items (Abb. 7b) erhielten wir hohe Zustimmungen für SI1, SI5 und SI6, jeweils mit einem Median von $\mathrm{m}=6$. Der Median von SI4 liegt bei $\mathrm{m}=5$, wobei die Abstimmungsergebnisse zwischen 4 (teilweise Zustimmung) und 7 (starke Zustimmung) schwanken. Der Median von SI2 liegt ebenfalls bei $\mathrm{m}=5$ und damit über dem Skalenwert der teilweisen Zustimmung, zeigt aber eine größere Schwankung auf als SI4 (Skalenwerte 2 bis 7). Dies kommt dadurch zustande, dass zwei Befragte unterhalb des Skalenwertes der teilweisen Zustimmung abgestimmt haben. Der Median von SI3 liegt bei $\mathrm{m}=4$ (teilweise Zustimmung). Auch hier schwanken die Abstimmungsergebnisse recht stark zwischen den Skalenwerten 3 und 7. Betrachtet man nur die Mediane, so zeigen SI1, SI2, SI4, SI5 und SI6 eine Bewertung oberhalb der teilweisen Zustimmung. Insbesondere die hohe Bewertung von SI6 (Gesamtnutzen) mit $m=6$ kann als Bestätigung für die allgemeine Nützlichkeit der Taxonomie angesehen werden. Diese Interpretation wird auch durch den Boxplot der Summenscores der wahrgenommenen Nützlichkeit gestützt, ermittelt aus der Summe der Skalen-Items der Fragebogen-Rückmeldungen (Abb. 7c). Hier liegt auf einer Skala von 7-42 der Mittelwert bei 32,5 und der Median bei 31,5 .

Aufgrund der großen Schwankungen in den Abstimmungsergebnissen für SI2 und SI3 haben wir die Experten erneut kontaktiert und ihnen die Möglichkeit geboten, Ergebnisse unterhalb des Skalenwertes von 4 zu begründen, mit dem Ziel, mögliche Anpassungspotenziale für unsere Taxonomie abzuleiten. Drei Teilnehmer antwor- 

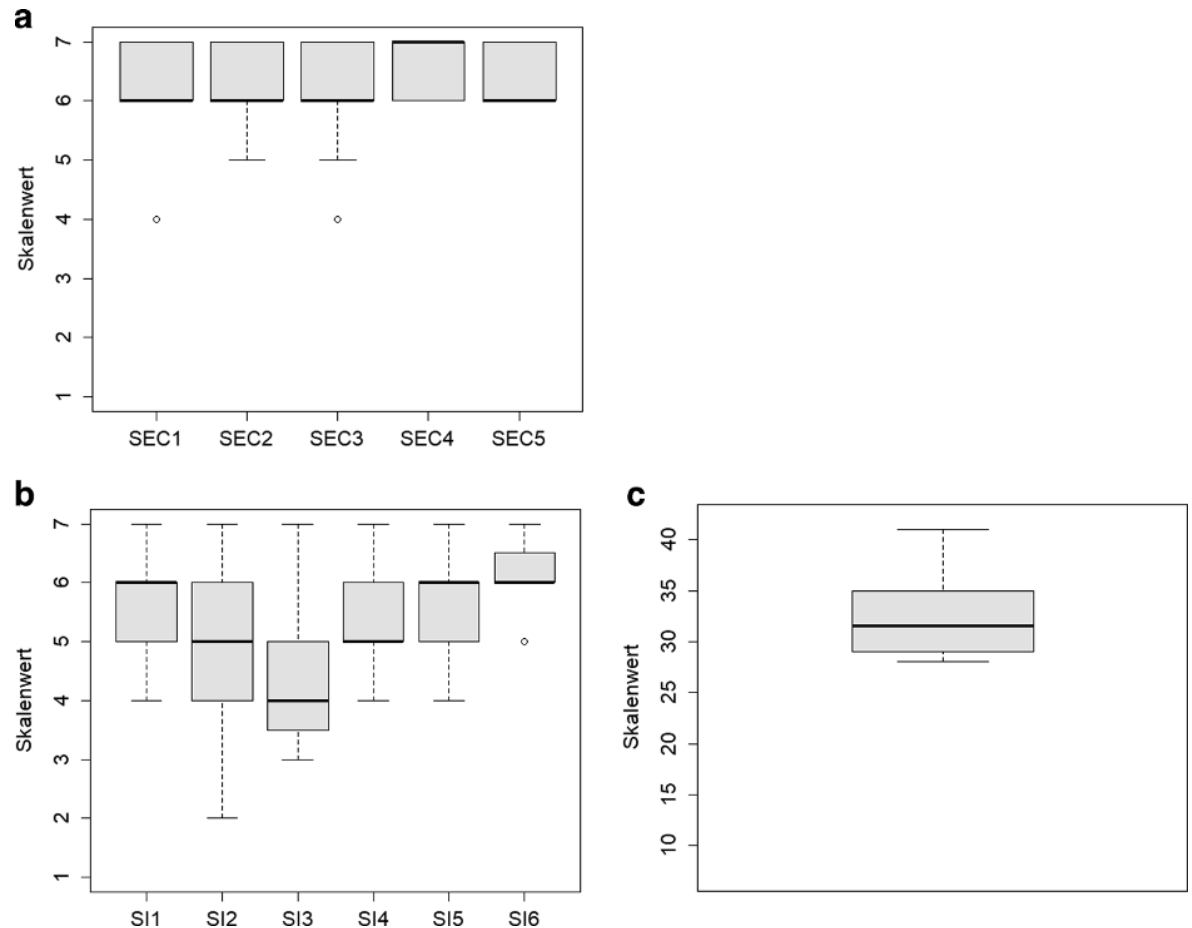

Abb. 7 Boxplots der Evaluationsergebnisse. a Subjektive Ending Conditions (SEC), b Skalen-Items der wahrgenommenen Nützlichkeit (SI), c Summenscore der Skalen-Items. (SEC1 Prägnanz, SEC2 Robustheit, SEC3 Vollständigkeit, SEC4 Erweiterbarkeit, SEC5 Erklärkraft, SI1 Schnelligkeit, SI2 Performanz, SI3 Produktivität, SI4 Effektivität, SI5 Einfachheit, SI6 Gesamtnutzen)

teten auf diese Folgefrage. Anhand der Antworten zeigte sich, dass der Einsatz von Taxonomien in der Praxis bisher kaum verbreitet ist, weshalb es für Praktiker insbesondere schwierig ist, deren Auswirkungen auf die Arbeitsleistung (SI2) und Produktivität (SI3) zu beurteilen. Unserer Meinung nach schränkt dieses Feedback die Nützlichkeit unserer Taxonomie insgesamt jedoch nicht ein. Dennoch wollen wir das wertvolle Feedback in zukünftiger Forschung nutzen, um einen engeren Transfer zwischen Wissenschaft und Praxis herzustellen, z. B. im Rahmen von sinnvollen Anwendungsfällen unserer Taxonomie.

\section{Anwendung der Taxonomie}

Um das Feedback der Evaluationsteilnehmer im vorherigen Kapitel aufgreifen möchten wir nun die Anwendbarkeit unserer Taxonomie demonstrieren. Einerseits kann die Taxonomie genutzt werden, um bestehende ARS zu kategorisieren. Dies haben wir exemplarisch für die in Kapitel 2 eingeführten Beispiele während der Erläuterung der Taxonomie in Kapitel 4 durchgeführt. Die Einordnung von ARS in die Taxonomie erlaubt es einerseits, ein ARS wirkungsvoll mit einem bekannten Vokabular zu 
beschreiben und macht es somit einfacher, dieses zu verstehen und darüber zu kommunizieren, bspw. im Zuge wissenschaftlicher Debatten. Ebenfalls haben wir die, in der strukturierten Literaturrecherche identifizierten, 142 ARS in die Taxonomie eingeordnet. Die Betrachtung der aggregierten Klassifizierungen ermöglicht einen wirkungsvollen Überblick über den aktuellen Forschungsstand und erleichtert die Analyse. Die Häufigkeit und Korrelation des Auftretens von Charakteristika ermöglicht die einfachere Identifikation von Trends, Best Practices und Forschungslücken innerhalb der Schnittmenge von AR, Workflows und WfMS.

Um dies zu demonstrieren, diskutieren wir im Folgenden zwei exemplarische Erkenntnisse aus der Analyse der 142 in die Taxonomie eingeordneten ARS. Erstens zeigt sich, dass sich die meisten der analysierten ARS auf spezifische Anwendungsszenarien konzentrieren und nur einen begrenzten, spezialisierten Funktionsumfang bieten. Wir halten es jedoch für wahrscheinlich, dass bedeutende Herausforderungen, Forschungsansätze und -fragen, bspw. in Bezug auf Designwissen, SoftwareArchitektur und User Experience, identifiziert werden könnten, wenn viele verschiedene Unterstützungsfunktionen integriert und mehrere Anwendungsszenarien mit einem einzigen ARS adressiert werden würden. Die zweite Erkenntnis ist, dass es eine Gelegenheit zu geben scheint, Konzepte aus dem Workflow-Management systematisch mit der AR-Forschung zu integrieren. Einerseits ist festzustellen, dass 10 der analysierten ARS eine formale Modellierung für die logische Struktur der AR-Inhalte nutzen und somit implizit auch eine Workflow-Engine, um diese zu interpretieren. Zusätzlich unterstützten viele ARS bereits einige Workflow-Management-Funktionen, wie die Auswertung dieser Dimension zeigt (vgl. Abb. 6). In Bezug auf die Workflow-Aufgaben-Unterstützung ist festzustellen, dass eine Vielzahl von Ansätzen existieren und insbesondere auch Ansätze, welche aus dem Workflow-Management bekannt sind, bspw. die Änderung des Workflow-Pfads aufgrund von Kontextinformationen oder die Empfehlung des nächstbesten Prozessschrittes (Prozess-Präskription). Andererseits jedoch verwendet kein ARS eine Standardmodellierungssprache wie bspw. den de facto Standard Business Process Model and Notation (BPMN) und in keiner ARS-Beschreibung wurde explizit eine WorkflowEngine oder ein WfMS zur Verarbeitung dieser Workflow-Modelle erwähnt. Die aus dem WfMS Referenzmodell der Workflow Management Coalition (1995) bekannten Workflow-Management Funktionen werden von keinem ARS vollständig adressiert und Workflow-Aufgaben-Unterstützungen, welche auf formalen Workflow-Modellen basieren, werden von nur wenigen ARS angeobten. Insgesamt sind im Stateof-the-Art also bereits viele Ansätze und Konzepte aus der Workflow-ManagementDomäne vorhanden, eine systematische Integration mit der AR-Domäne ist bislang jedoch nicht erfolgt. Es scheint somit eine Gelegenheit für eine solche Integration zu geben, bspw. konzeptionell zur Verbindung von Referenzarchitekturen oder gestalterisch zur Schaffung von Designwissens über ARS.

Um Forschungslücken zu adressieren kann die Taxonomie auch genutzt werden, um in einer strukturierten Weise neue ARS zu entwerfen. Dies haben wir im Rahmen unserer eigenen Forschung für zwei ARS bereits umgesetzt.

Erstens haben wir so das ARS für das laufende BMBF-Verbundprojekt Augmented Living Spaces (ALiS) entworfen, welches mittels AR-Inhalten zum Erhalt der Selbstständigkeit kognitiv eingeschränkter Menschen in ihren eigenen Wohnräumen 
beitragen soll. Dieses ARS basiert auf spatialer AR, sodass die Nutzer keine Geräte tragen müssen, um die AR-Inhalte zu nutzen (Bimber und Raskar 2005). Stattdessen werden visuelle und akustische Inhalte mit Projektoren und Lautsprechern wiedergegeben. Es adressiert somit die relative Unterrepräsentation der spatialen AR in den 142 analysierten ARS, von welchen lediglich 15 Projektoren und 3 stationäre Lautsprecher nutzen. In Kombination mit Eye-Tracking (6), Erkennung von Körperhaltungen (4) und allgemeiner Positionsverfolgung, z. B. über RFID (3), wollen wir eine nahtlose und dennoch individuelle Unterstützung für Benutzer an vordefinierten Orten erzeugen. Es soll Echtzeitdaten (17) von Umgebungssensoren nutzen, um dann bspw. animierte (23) und akustische (14) Warnhinweise zu geben, z. B. bei heißen Herdplatten. Routing-Funktionen (6) könnten zu verlorenen Gegenständen oder bestimmten Räumen führen. Diese verschiedenen AR-Unterstützungsfunktionen werden als Workflows modelliert und mittels des WfMS Camunda ausgeführt, welches die übrigen Sensoren, Datenspeicher, Datenverarbeitung und Ausgabegeräte integriert. Die Unterstützungsworkflows werden in einem, in Entwicklung befindlichen Erweiterungsdialekt der BPMN modelliert, welche speziell die Anforderungen von ARS adressieren. Die Nutzung eines WFMS ermöglicht die schnelle Modellierung und Implementierung in einer Standardnotation, die einfache Integration verschiedener Systeme (bspw. Unity für AR; NodeRED für Sensoren) sowie auch das effektive Management der Workflows. Zudem können die Workflow-Logs mit Standardwerkzeugen analysiert werden.

Zweitens läuft an der Professur für Wirtschaftsinformatik, insb. Betriebliches Informationsmanagement der Martin-Luther-Universität Halle-Wittenberg das Forschungsprojekt HoloWFM, welches die Lücke bezüglich WfMS-Management-Funktionalitäten in den 142 analysierten ARS adressiert. Das so entstehende ARS wird gemäß dem bekannten WfMS Referenzmodell der Workflow Management Coalition (1995) alle WfMS Front End-Funktionen, im Sinne einer Workflow Client Application implementieren und damit die Dimension ,Workflow-Management“ der Taxonomie erweitern. Dieses ARS solle den Nutzern ermöglichen effektiv, effizient und benutzerfreundlich mit einem WfMS zu interagieren und gleichzeitig von anderen AR-basierten Funktionen zur Unterstützung der Workflow-Ausführung zu profitieren, wodurch zusätzliche Geräte überflüssig würden und Medienbrüche vermieden werden können. Während das Forschungsprojekt auf die Schaffung von Designwissen angelegt ist, wird ein HoloWFM-Prototyp die API des WfMS Camunda nutzen, um die Funktionalität des AR-Front Ends zu demonstrieren und das Gesamtprojekt zu evaluieren.

\section{Zusammenfassung und Ausblick}

In diesem Beitrag wurde eine Taxonomie zur Charakterisierung von ARS vorgestellt, welche die Workflow-Ausführung unterstützen. Durch eine strukturierte Literaturrecherche wurden 142 relevante ARS identifiziert und aus deren Eigenschaften eine Taxonomie bestehend aus 14 Dimensionen und 83 Charakteristiken abgeleitet. Die Taxonomie wurde abschließend mit einer Bewertung der wahrgenommenen Nütz- 
lichkeit (Davis 1989) positiv evaluiert. Zwei mit der Taxonomie entwickelte ARS wurden als Beispiele für die Anwendbarkeit vorgestellt.

Für eine adäquate Interpretation der Ergebnisse sollten die folgenden Limitationen berücksichtigt werden. Erstens basierte die Taxonomie auf einer strukturierten Literaturrecherche und umfasste nur Charakteristiken, die in den analysierten ARS identifiziert wurden. Zweitens ist eine inhärente Schwäche jeder Taxonomie-Entwicklung die Subjektivität der zugrundeliegenden Designentscheidungen. Wie der Vergleich mit verwandten Beiträgen gezeigt hat, sind andere Einteilungen der Charakteristiken möglich (Kalawsky et al. 2000; Klinker et al. 2018; Milgram und Kishino 1994; Wang et al. 2013). Die getroffenen Designentscheidungen werden jedoch durch die evaluierten objektiven und subjektiven Endbedingungen von Nickerson et al. (2013) und die Evaluation unserer Taxonomie mit Experten untermauert. Drittens waren die Beschreibungen der analysierten ARS im Kontext der Taxonomie oft schwer zu interpretieren, wodurch einige ARS falsch klassifiziert sein können.

Eine Taxonomie basiert auf den aktuell existierenden Objekten. Die hier vorgestellte Taxonomie thematisiert mit Augmented Reality und Workflow-ManagementSystemen zwei sich schnell entwickelnde Technologien. Somit wird diese Taxonomie höchstwahrscheinlich in Zukunft angepasst werden müssen, um die technologischen Fortschritte zu reflektieren. Für Augmented Reality könnten dies neue sensorische Kanäle für die Ein- und Ausgabe sein, welche teilweise bereits in der Literatur identifiziert, jedoch in den untersuchten ARS nicht festgestellt wurden. Für das Management von Workflows ist es wahrscheinlich, dass neue ARS sich dem, in der bekannten Referenzarchitektur der Workflow Management Coalition postulierten, Interaktionsspektrum annähern. Die Workflow-Aufgaben-Unterstützung könnte besonders durch neue Entwicklung im Maschinellen Lernen profitieren. Zuletzt wird auch der weitere Praxiseinsatz Erkenntnisse generieren, die in einer Weiterentwicklung der Taxonomie einfließen könnten.

Zusatzmaterial online Zusätzliche Informationen sind in der Online-Version dieses Artikels (https://doi. org/10.1365/s40702-021-00831-y) enthalten.

Funding Open Access funding enabled and organized by Projekt DEAL.

Open Access Dieser Artikel wird unter der Creative Commons Namensnennung 4.0 International Lizenz veröffentlicht, welche die Nutzung, Vervielfältigung, Bearbeitung, Verbreitung und Wiedergabe in jeglichem Medium und Format erlaubt, sofern Sie den/die ursprünglichen Autor(en) und die Quelle ordnungsgemäß nennen, einen Link zur Creative Commons Lizenz beifügen und angeben, ob Änderungen vorgenommen wurden.

Die in diesem Artikel enthaltenen Bilder und sonstiges Drittmaterial unterliegen ebenfalls der genannten Creative Commons Lizenz, sofern sich aus der Abbildungslegende nichts anderes ergibt. Sofern das betreffende Material nicht unter der genannten Creative Commons Lizenz steht und die betreffende Handlung nicht nach gesetzlichen Vorschriften erlaubt ist, ist für die oben aufgeführten Weiterverwendungen des Materials die Einwilligung des jeweiligen Rechteinhabers einzuholen.

Weitere Details zur Lizenz entnehmen Sie bitte der Lizenzinformation auf http://creativecommons.org/ licenses/by/4.0/deed.de. 


\section{Literatur}

Aldaz G, Shluzas LA, Pickham D, Eris O, Sadler J, Joshi S, Leifer L (2015) Hands-free image capture, data tagging and transfer using Google Glass: a pilot study for improved wound care management. PLoS ONE 10(4):e121179

Andersen RS, Bogh S, Moeslund TB, Madsen O (2016) Task space HRI for cooperative mobile robots in fit-out operations inside ship superstructures. In: RO-MAN 2016 - Proceedings of the 25th IEEE International Symposium on Robot and Human Interactive Communication. IEEE, New York, S 880-887

Arroyave-Tobón S, Osorio-Gómez G, Cardona-McCormick JF (2015) AIR-MODELLING: A tool for gesture-based solid modelling in context during early design stages in AR environments. Comput Ind 66:73-81

Azuma R, Baillot Y, Behringer R, Feiner S, Julier S, MacIntyre B (2001) Recent advances in augmented reality. Ieee Cga 21(6):34-47

Bailey KD (1994) Typologies and taxonomies. An introduction to classification techniques. SAGE, Thousand Oaks

Berkemeier L, Zobel B, Werning S, Hinrichs U, Ickerott I, Thomas O (2019) Introducing smart glasses to logistics services providers: a single case study from a wholesale warehouse. In: Digitale Dienstleistungsinnovationen. Springer, Berlin Heidelberg, S 307-328

Bimber O, Raskar R (2005) Spatial augmented reality. CRC Press, Boca Raton

Blanco-Novoa O, Fernandez-Carames TM, Fraga-Lamas P, Vilar-Montesinos MA (2018) A practical evaluation of commercial industrial augmented reality systems in an industry 4.0 shipyard. IEEE Access 6:8201-8218

vom Brocke J, Simons A, Niehaves B, Riemer K, Plattfaut R, Cleven A (2009) Reconstructing the giant: on the importance of rigour in documenting the literature search process. In: ECIS 2009. Verona, ITA

Chicaiza EA, de La Cruz EI, Andaluz VH (2018) Augmented reality system for training and assistance in the management of industrial equipment and instruments. In: Bebis G et al (Hrsg) Advances in visual computing. ISVC 2018. Springer, Cham, S 675-686

Craig AB (2013) Understanding augmented reality. Concepts and applications. Elsevier, San Diego

Davis FD (1989) Perceived usefulness, perceived ease of use, and user acceptance of information technology. MISQ 13(3):319-340

Dünser A, Billinghurst M, Wen J, Lehtinen V, Nurminen A (2012) Exploring the use of handheld AR for outdoor navigation. Comput Graph 36(8):1084-1095

Erkoyuncu JA, Del Amo IF, Dalle Mura M, Roy R, Dini G (2017) Improving efficiency of industrial maintenance with context aware adaptive authoring in augmented reality. Cirp Ann 66(1):465-468

Evans G, Miller J, Iglesias Pena M, MacAllister A, Winer E (2017) Evaluating the Microsoft HoloLens through an augmented reality assembly application. In: SPIE Defense+Security, Proceedings SPIE, Bd. 10197 (Article 101970V)

Feng S, Caire R, Cortazar B, Turan M, Wong A, Ozcan A (2014) Immunochromato-graphic diagnostic test analysis using Google Glass. Acs Nano 8(3):3069-3079

Ferrari V, Viglialoro RM, Nicoli P, Cutolo F, Condino S, Carbone M, Siesto M, Ferrari M (2016) Augmented reality visualization of deformable tubular structures for surgical simulation. MRCAS 12(2):231-240

Fiorentino M, Uva AE, Gattullo M, Debernardis S, Monno G (2014) Augmented reality on large screen for interactive maintenance instructions. Cancer Immunol Immunother 65(2):270-278

Gröger C, Schwarz H, Mitschang B (2014) Prescriptive analytics for recommendation-based business process optimization. BIS 2014(176):25-37

Hanson R, Falkenström W, Miettinen M (2017) Augmented reality as a means of conveying picking information in kit preparation for mixed-model assembly. Comput Ind Eng 113:570-575

Henderson SJ, Feiner SK (2011) Augmented reality in the psychomotor phase of a procedural task. In: ISMAR 2011, S 191-200

Hevner A, March ST, Park J, Ram S (2004) Design science in information systems research. MISQ 28(1):75

Hofmann C, Staehr T, Cohen S, Stricker N, Haefner B, Lanza G (2019) Augmented go \& see: an approach for improved bottleneck identification in production lines. Procedia Manuf 31:148-154

Hou L, Wang X, Bernold L, Love P (2013) Using animated augmented reality to cognitively guide assembly. J Comp Civ Eng 27(5):439-451. https://doi.org/10.1061/(ASCE)CP.1943-5487.0000184

Hwang W, Salvendy G (2010) Number of people required for usability evaluation. Commun ACM 53(5):130-133 
Jetter J, Eimecke J, Rese A (2018) Augmented reality tools for industrial applications: What are potential key performance indicators and who benefits? CiHB 87:18-33

Kalawsky RS, Stedmon AW, Hill K, Cook CA (2000) A taxonomy of technology: defining augmented reality. Proc Hum Factors Ergonomics Soc Annu Meet 44(5):507-510

Klinker K, Berkemeier L, Zobel B, Huck-Fries V, Wüller H, Wiesche M, Remmers H, Thomas O, Krcmar H (2018) Structure for innovations: a use case taxonomy for smart glasses in service processes. In: MKWI 2018, S 1599-1610

Krcmar H (2006) Information management. Springer, Berlin

van Krevelen D, Poelman R (2010) A survey of augmented reality technologies, applications and limitations. IJVR 9(2):1-20

Lahanas V, Loukas C, Smailis N (2015) A novel augmented reality simulator for skills assessment in minimal invasive surgery. Surg Endosc 29(8):2224-2234

Lampen E, Teuber J, Gaisbauer F, Bär T, Pfeiffer T, Wachsmuth S (2019) Combining simulation and augmented reality methods for enhanced worker assistance in manual assembly. CIRP 81:588-593

Liebmann F, Roner S, von Atzigen M, Scaramuzza D, Sutter R, Snedeker J, Farshad M, Fürnstahl P (2019) Pedicle screw navigation using surface digitization on the Microsoft HoloLens. IJCARS 14(7):1157-1165

Limbu B, Vovk A, Jarodzka H, Klemke R, Wild F, Specht M (2019) WEKIT.One: a sensor-based augmented reality system for experience capture and re-enactment. In: EC-TEL 2019, S 158-171

Liu H, Auvinet E, Giles J, Rodriguez y Baena F (2018) Augmented reality based navigation for computer assisted hip resurfacing: a proof of concept study. Ann Biomed Eng 46(10):1595-1605. https://doi. org/10.1007/s10439-018-2055-1

MacWilliams A, Reicher T, Klinker G, Bruegge B (2004) Design patterns for augmented reality systems. In: UI-CADUI*04

Makris S, Pintzos G, Rentzos L, Chryssolouris G (2013) Assembly support using AR technology based on automatic sequence generation. CIRP 62(1):9-12

March ST, Smith GF (1995) Design and natural science research on information technology. Decis Support Syst 15(4):251-266

Metzger D, Niemöller C, Jannaber S, Berkemeier L, Brenning L, Thomas O (2018) The next generation-design and implementation of a smart glasses-based modelling system. EMISAJ 13(18):1-25

Milgram P, Kishino F (1994) A taxonomy of mixed reality visual display. IEICE Trans Inf \& Syst E77D(12):1321-1329

Neges M, Wolf M, Abramovici M (2015) Secure access augmented reality solution for mobile maintenance support utilizing condition-oriented work instructions. Procedia Cirp 38:58-62

Nickerson RC, Varshney U, Muntermann J (2013) A method for taxonomy development and its application in information systems. EJIS 22(3):336-359

Pries-Heje J, Baskerville R, Venable JR (2008) Strategies for design science research evaluation. In: ECIS 2008

Seiger R, Kühn R, Korzetz M, Aßmann U (2021) HoloFlows: modelling of processes for the Internet of things in mixed reality. Softw Syst Model 20:1465-1489

Szopinski D, Schoormann T, Kundisch D (2019) Because your taxonomy is worth it: towards a framework for taxonomy evaluation. In: ECIS 2019. Verona, ITA

Wang X, Kim MJ, Love PE, Kang S-C (2013) Augmented reality in built environment: classification and implications for future research. Auto Constr 32:1-13

Wang X, Ong SK, Nee A (2016) Multi-modal augmented-reality assembly guidance based on bare-hand interface. Adv Eng Inf 30(3):406-421

Webster J, Watson RT (2002) Analyzing the past to prepare for the future: writing a literature review. MISQ 26(2):xiii-xxiii

Workflow Management Coalition (1995) The Workflow reference model 